\title{
A Novel Planar Differential Koch Fractal Eddy Current Probe with Parallel Wound Topological Structure
}

\author{
Guolong Chen $\mathbb{D}^{1},{ }^{1}$ Zheng Cao, ${ }^{1}$ and Weimin Zhang $\mathbb{D}^{2}$ \\ ${ }^{1}$ School of Mechanical and Electrical Engineering, Lanzhou University of Technology, Lanzhou 730500, China \\ ${ }^{2}$ School of Mechanical Engineering, Beijing Institute of Technology, Beijing 100081, China \\ Correspondence should be addressed to Weimin Zhang; zhangwm@bit.edu.cn
}

Received 23 October 2020; Revised 19 January 2021; Accepted 26 April 2021; Published 17 June 2021

Academic Editor: Abdellah Touhafi

Copyright (C) 2021 Guolong Chen et al. This is an open access article distributed under the Creative Commons Attribution License, which permits unrestricted use, distribution, and reproduction in any medium, provided the original work is properly cited.

\begin{abstract}
Flexible planar eddy current probes are widely used to detect conductive components with complex surface. In this study, topological transformation is applied to design a differential Koch coil exciting eddy current probe. Two kinds of Koch exciting coils, Koch $\mathrm{A}$ and Koch $B$, were obtained by topological transformation from a three-dimensional differential exciting eddy current probe. Finite element model simulation is conducted to visualize the differences of eddy current distributions induced by the probes and get the defect signal. A detailed comparison is made among the two kinds of Koch eddy current probes and a circular eddy current probe by experiments. The experiments demonstrate that the sensitivity of the Koch A eddy current probe is higher than that of another two probes for detecting the defect which is shorter than the size of the probe. This work provides a novel method for improving the performance of eddy current probes in the coil structure design.
\end{abstract}

\section{Introduction}

As the beginning of obtaining information from eddy current (EC) testing (ECT) instruments, EC probes directly affect the performance of instruments. Hence, how to improve the performance of the EC probes has always been one of the research hotspots in this field. So far, there are mainly two approaches to enhance the performance of EC probes: the waveform of exciting currents and the structure of probe's coils.

With the further understanding of the ECT, the waveform of the exciting current of the EC probe has gone through a process of development from single-frequency to multifrequency and then from simple to complex waveform. The single-frequency sine waveform was employed as an exciting current of EC probes in the earliest EC testing. In order to enrich the information obtained by EC probes, suppress lift-off noise, and improve permeable depth of eddy currents, the waveforms such as dual-frequency [1], multifrequency [2], swept-frequency, chirp [3], pulse $[4,5]$, and pulse-modulated sine wave $[6,7]$ were used as the waveforms of an exciting current for EC probes.
On the other hand, the way to enhance the performance of EC probes from improving structures is mainly reflected in the following methods: the probe element has been developed from a single coil to multicoil such as the differential exciting EC probes which adopt two exciting coils and the differential pick-up EC probes which adopt two pick-up coils [8] and the rotating field EC probes which adopt two exciting currents with the same frequency and the $90^{\circ}$ phase difference fed into two perpendicular driving traces $[9,10]$; the number of elements has been developed from a singlechannel towards array [11]; the detecting elements of probes have been developed from coils to magnetosensors with high sensitivity such as hall, giant magnetoresistance (GMR), tunnel magnetoresistance (TMR), and other magnetosensors $[11,12]$; the shape of coils has been developed from conventional line [13], circle, and spiral to special-shaped structure such as gradient EC probes [14]; and the dimension of probes has been developed from a three-dimensional rigid structure to a two-dimensional flexible structure $[15,16]$.

Usually, the EC testing can be explained as an interaction between the ECs and the tested parts, and the physical properties or geometric properties of the tested parts are 
modulated in the EC distribution and then embodied in a secondary magnetic field. Subsequently, this kind of information is obtained by the pick-up element to achieve the testing goal. From the perspective of the Fourier transform, a complex exciting current waveform consists of different frequency components, and the permeable depth of EC is relevant to the frequency $[6,7]$. Therefore, the complex exciting current waveform is used to increase the ability to modulate the information in depth direction of the tested components.

For detecting the parts with complex shape like cranks and aeroengine blades, flexible planar sensors that can reconstruct the surface to suit the surface of the tested parts were put forward. In this way, the shortcomings, which include the area that is hard to reach and the sensors that are easily influenced by lift-off noise in the conventional rigid sensors, can be improved [17]. Daura et al. [18] proposed using the wireless power transfer to efficiently acquire the defect signal of the transmitter-receiver flexible printed coil. She et al. [19] proposed the flexible floral eddy current probe, which is a differential exciting eddy current probe to suppress the lift-off noise and to detect the flaws in the metal plate. Zhang et al. [20] proposed a novel flexible array eddy current probe with a three-phase alternative current to excite the three exciting coils. At the same time, with the development of the flexible circuit board technology, it is possible to explore the flexible planar sensors with a complex-shaped coil structure [21]. This method is aimed at improving the EC distribution and modulating the defect information of tested parts into the output signals better.

Chen et al. adopted the fractal geometry Koch curves to design the exciting coil of the EC probes for the first time [22]. In that study, the Koch curves were used as the exciting coil and the circular spiral as the pick-up coil. However, the coil structure has disadvantages in the following aspects:

(1) The probe is an absolute EC probe which is more susceptible to the lift-off noise

(2) Its absolute structure determines that the defect information occupies an extremely small proportion in its output signals. Therefore, it is difficult to extract the microcrack information in the output signal

Given that there are two drawbacks mentioned above, this work proposes a differential Koch coil exciting planar EC probe. To start with, the method of the coil connection of the conventional three-dimensional differential exciting EC probes is analyzed. The topological transformation is used to analyze the two connection ways of the differential exciting coils of the EC probes. Then, series-wound structure and parallel-wound topological structure are obtained. Afterwards, with the parallel-wound topological structure selected, two Koch coil exciting EC probes are designed by topological transformation. To compare them with a circular exciting coil, a circular coil EC probe with the same size and topological structure is designed to conduct a comparative experiment. Finally, a comparative study for the probes is carried out via the finite element model (FEM) simulation and experiments.

\section{Probe Design}

In this section, the principle of two kinds of differential EC probes will be introduced. Then, the topological structures of exciting coils of the differential exciting EC probes will be analyzed. Then, two topological structures, which are series-wound and parallel-wound topological structure, will be obtained. In this article, the ideas of parallel-wound topological structure and Koch curve will be fused to design the exciting coil of a planar EC probe, while the series-wound topological structure will be reported in another article. At the end of this section, the geometric details of the probes will be presented.

In the EC NDT, the main advantage of the differential EC probe can easily suppress the lift-off noise by the coil structure design [23]. In the structural design, EC probes include differential exciting type and differential pick-up type, as shown in Figure 1. For the differential exciting EC probe, in theory, the magnetic field or the magnetic flux linkage built by exciting coils in the pick-up element is cancelled to each other, when the probe is positioned in the air and on the ideal tested sample which ensures that exciting coils have the same lift-off distance from the sample. However, for the differential pick-up probe, two or more pick-up elements are arranged in the probe, and the output signals are cancelled to each other in the same condition. In this work, the differential exciting EC probe was focused on. In general, a differential exciting planar EC probe can be designed by connecting the two coils. However, for the planar EC probe, the coils are placed in a two-dimensional space, which is different from a traditional EC probe arranged in the three-dimensional space. Thus, there is constraint of the space for designing the coils of the planar EC probe. In view of this situation, the method of the exciting coil connection needs to be analyzed by using topology.

In topology, when a graph is transformed into another graph by a continuous and slow change, the two graphs have the same topological structure [24]. This transformation method is used in the differential exciting EC probe. The two exciting coils of the differential exciting EC probe can be connected in series and in parallel. When two exciting coils are in series as shown in Figure 2(a), the exciting current flows into node $A$ and out from node $B$, and the exciting current is the same at every point of the two coils. If the two coils connected in series are slowly stretched, the coils can be transformed into a line segment with two nodes, as shown in Figure 2(a). Thus, the two coils in series and the line segment are of the same topological structure, and this is named the series-wound topological structure. However, if two exciting coils are connected in parallel, those can be stretched to another structure, which includes two line segments, a loop, and 4 nodes, as shown in Figure 2(b). For this structure, if the exciting current flows in node $A$, it will be divided into two streams at point $C$; then, the two streams of currents gather in node $D$ and flow out from $B$, as shown in Figure 2(b). The structure in Figure 2(b) is named the parallel-wound topological structure. The parallel-wound topological structure is selected to design the differential 


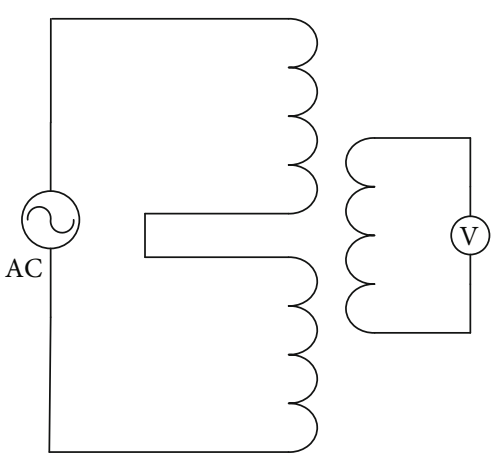

(a)

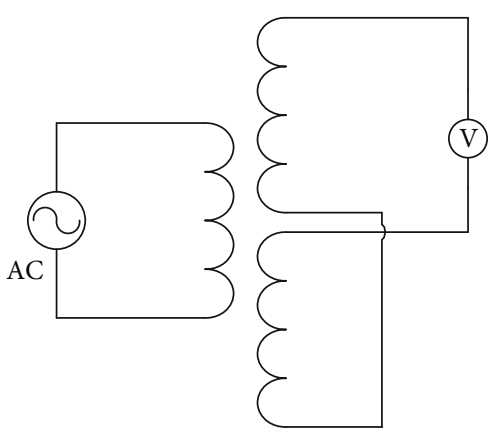

(b)

FIgure 1: Two kinds of differential EC probe: (a) differential exciting EC probe; (b) differential pick-up EC probe.

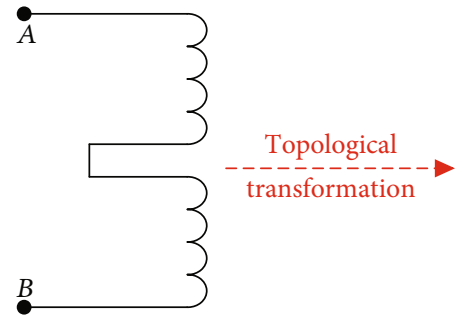

(a)

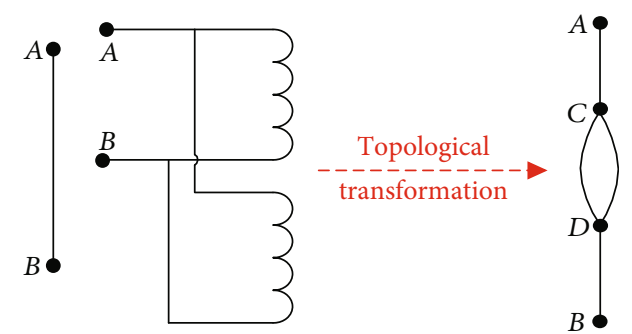

(b)

FIGURE 2: Topological transformation of the two kinds of connection of two exciting coils: (a) series-wound topological structure; (b) parallelwound topological structure.

exciting EC probe, but the series-wound topological structure will be presented in another work.

Combining the topological transformation, symmetry, and fractal geometry, three types of exciting coils are designed, as shown in Figure 3. The loop between nodes $C$ and $D$ is changed to a circle, as an exciting coil, as shown in Figure 3(b). Because circular coil is widely used to be an exciting coil, the exciting coil in Figure 3(a) is used for comparison. Then, for the EC distribution conditioning idea, the Koch curve is selected to design the exciting coil [19]. Because the Koch curve has two symmetrical axes, the loop between node $C$ and node $D$ in Figure 3(a) can be topologically transformed into two kinds of exciting coils in which the axis of the Koch curve overlaps the line between $A$ and $B$, as shown in Figures 3(c) and 3(d). The new exciting coils in Figures 3(c) and 3(d) are named Koch $A$ and Koch $B$, respectively. The three new exciting coils are symmetrical about line $A B$. If an exciting current is fed into one of the three exciting coils, the magnetic field in the center loop generated by the left part and right part in the center loop will be symmetrically distributed in magnitude but be opposite in direction, so the magnetic flux in the center loop is zero. This is the principle of the proposed differential exciting coils.

The three prototype probes are designed in a printed circuit board (PCB), as shown in Figure 4. Every probe includes an exciting coil and a pick-up coil, and the color of the exciting coil is red but that of the pick-up coil is blue. The exciting coils and the pick-up coils are arranged in the top layer and bottom layer of PCB whose thickness is $0.6 \mathrm{~mm}$, respectively. The loop of the exciting coil of the circular probe is a circle with $10 \mathrm{~mm}$ diameter, but that of Koch $A$ and $B$ is a second-order Koch curve, and the diameter of the circumcircle of the Koch curve is $10 \mathrm{~mm}$. The widths of the exciting and pick-up coils are $0.31 \mathrm{~mm}$ and $0.15 \mathrm{~mm}$, respectively. The exciting and pick coils are arranged on the top layer and the bottom layer of the PCB, respectively, and the centers of the exciting coil, the pick-up coil, and the PCB coincide.

\section{FEM Simulation}

In order to view the EC distributions induced by the probes and defect signals, 6 different FEMs are built and calculated by the AC/DC model of the commercial software COMSOL multiphysics. For every probe, two models are built; one has a perfect aluminum specimen, but the other has a specimen with an infinite defect which cut the specimen surface.

Every model includes three parts: air domain, aluminum with an infinite defect or no defect, and a probe including an exciting coil and a pick-up coil. The size of the air domain is $150 \mathrm{~mm} \times 100 \mathrm{~mm} \times 40 \mathrm{~mm}$. The size of the specimen is $120 \mathrm{~mm} \times 80 \mathrm{~mm} \times 6 \mathrm{~mm}$, and if a specimen includes an infinite defect on the top surface, the size of the slot defect will be $0.25 \mathrm{~mm} \times 80 \mathrm{~mm} \times 3 \mathrm{~mm}$. The geometric dimensioning of the exciting coil and the pick-up coil is identical to the prototype probes; the distance of the exciting coil and the pick-up coil from the top surface of the specimen is $0.5 \mathrm{~mm}$ and $0.65 \mathrm{~mm}$, respectively. The driver traces $A C$ and $B D$ in Figure 3 are extended to the boundary of the air domain. In every model, the exciting coil and the pick-up coil are ideal lines whose cross-sectional area is zero. The geometrical 


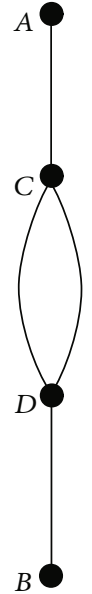

(a)

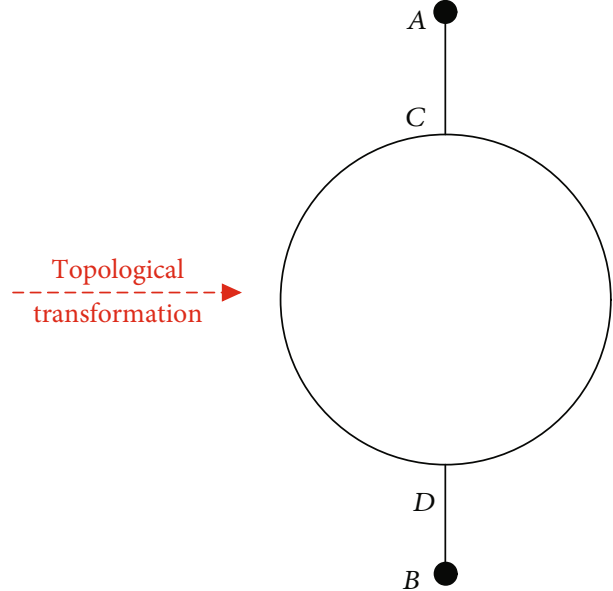

(b)

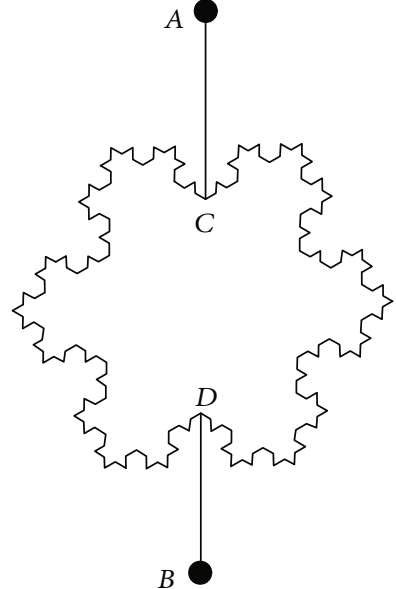

(c)

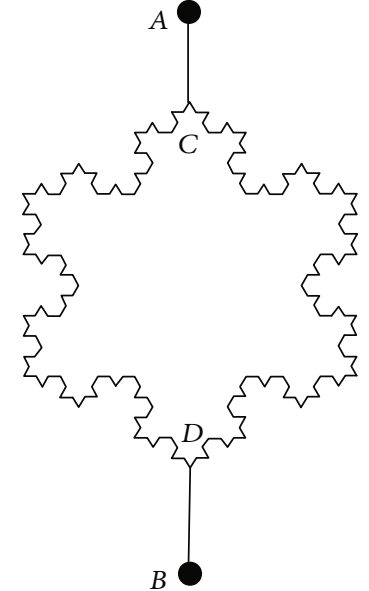

(d)

Figure 3: Topological transformation of parallel-wound topological structure: (a) topological; (b) circle; (c) Koch $A$; (d) Koch $B$.

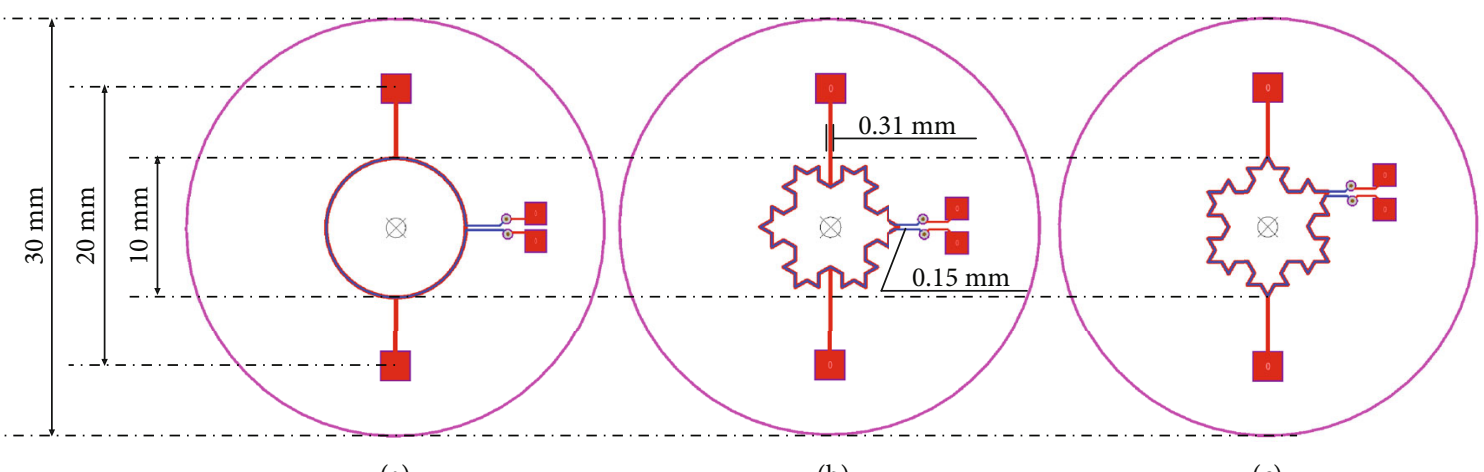

(a)

(b)

(c)

FIGURE 4: The drawing of the three types of the prototypes: (a) circle; (b) Koch A; (c) Koch $B$.

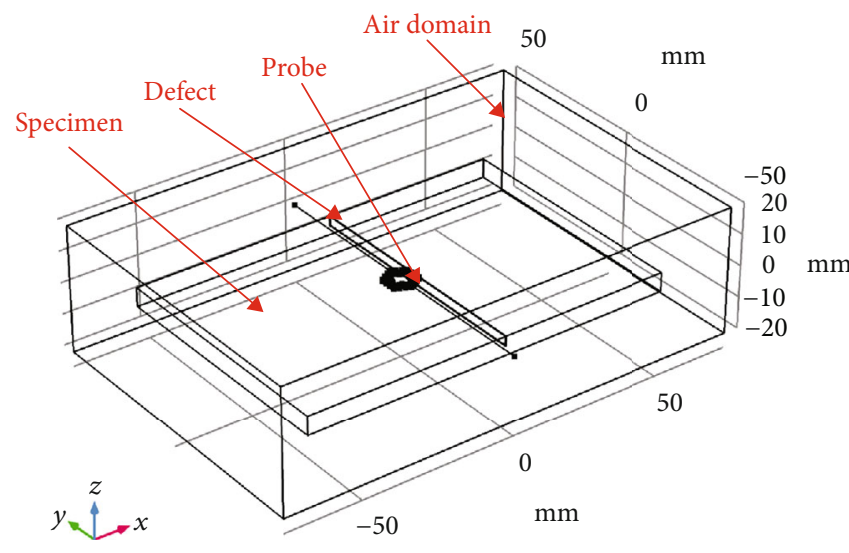

(a)

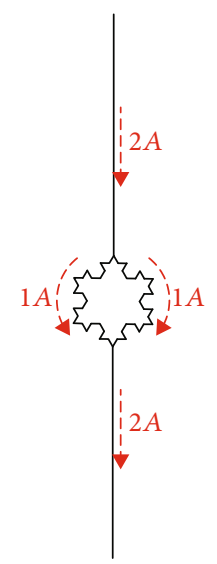

(b)<smiles>C1CCCCCC1</smiles>

(c)

Figure 5: The geometrical model with infinite defect of the Koch $B$ probe: (a) the entire model; (b) exciting coil; (c) pick-up coil. 


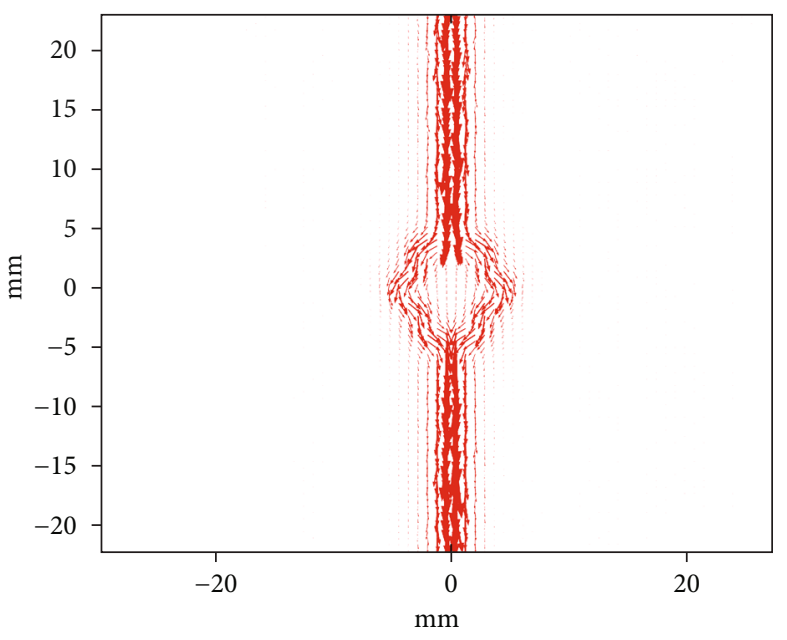

(a)

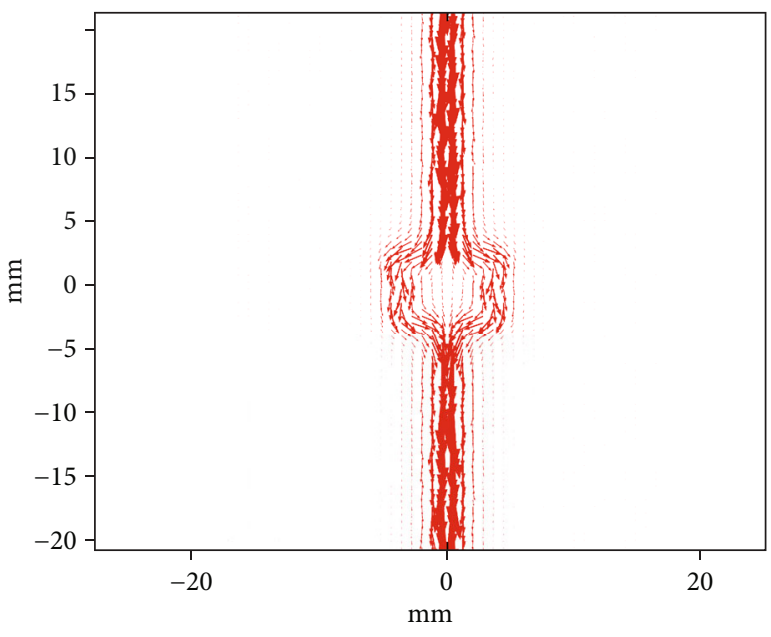

(b)

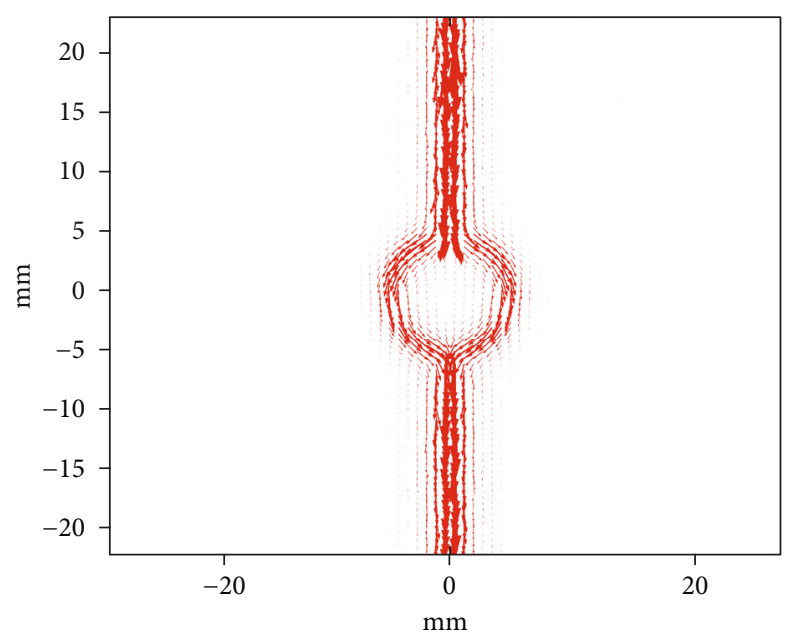

(c)

Figure 6: EC distributions on the upper surface of the specimen: (a) Koch A; (b) Koch B; (c) circle.

model with an infinite defect of the Koch $B$ probe is shown in Figure 5.

There are two materials selected in every model: air and aluminum. The relative permeability and relative permittivity are 1 for the two materials; the electrical conductivity of the air and the aluminum is $1 \mathrm{~S} / \mathrm{m}$ and $3.77 \times 10^{7} \mathrm{~S} / \mathrm{m}$, respectively.

All FEMs are calculated in the frequency domain. The exciting current is typed by the edge current. The exciting currents of the main roads which are $A C$ and $B D$ in Figure 3 are typed to $2 A$, but those of the branches of the exciting coil are typed to $1 A$, as shown in Figure 5(c). The physics control equations of the aluminum are as follows:

$$
\left\{\begin{array}{l}
\nabla \times \mathbf{H}=\mathbf{J}, \\
\mathbf{B}=\nabla \times \mathbf{A}, \\
\mathbf{E}=-j \omega \mathbf{A}, \\
\mathbf{J}=\sigma \mathbf{E}+j \omega \mathbf{D},
\end{array}\right.
$$

where $\mathbf{H}, \mathbf{B}, \mathbf{J}, \mathbf{A}, \mathbf{E}$, and $\mathbf{D}$ are magnetic field, magnetic flux density, current density, magnetic vector potential, electric field, and displacement density, respectively. The constitutive relations of isotropic material are as follows:

$$
\left\{\begin{array}{l}
\mathbf{B}=\mu_{0} \mu_{r} \mathbf{H}, \\
\mathbf{D}=\epsilon_{0} \epsilon_{r} \mathbf{E},
\end{array}\right.
$$

where $\mu_{0}, \mu_{r}, \epsilon_{0}$, and $\epsilon_{r}$ are permeability of air, relative permeability, permittivity of air, and relative permittivity, respectively. The boundary conditions, which are automatically set by software, are magnetic insulation and electric insulation:

$$
\left\{\begin{array}{l}
\mathbf{n} \times \mathbf{A}=\mathbf{O}, \\
\mathbf{n} \cdot \mathbf{J}=0,
\end{array}\right.
$$

where $\mathbf{n}$ is the vector perpendicular to the surfaces of the aluminum and the air domain. 


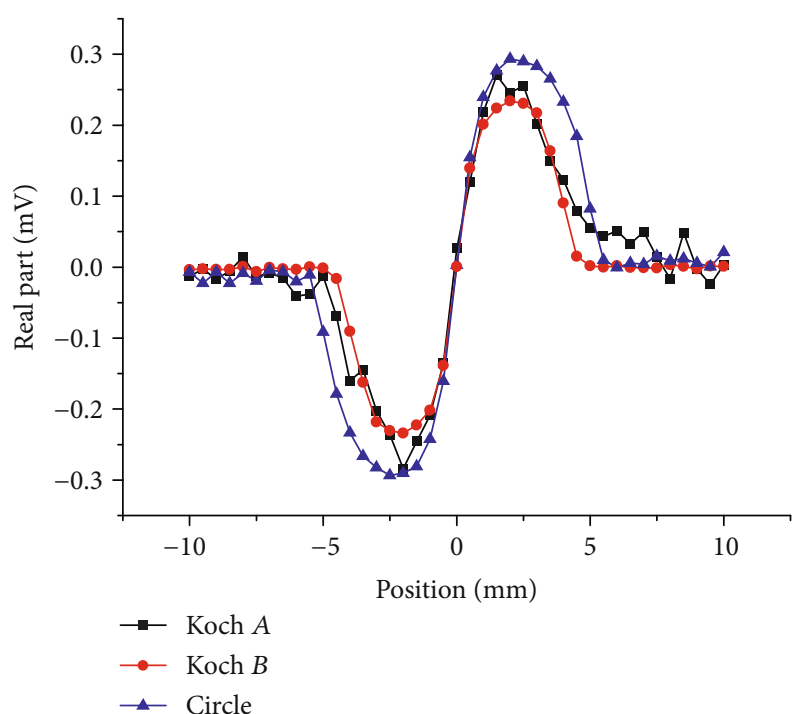

(a)

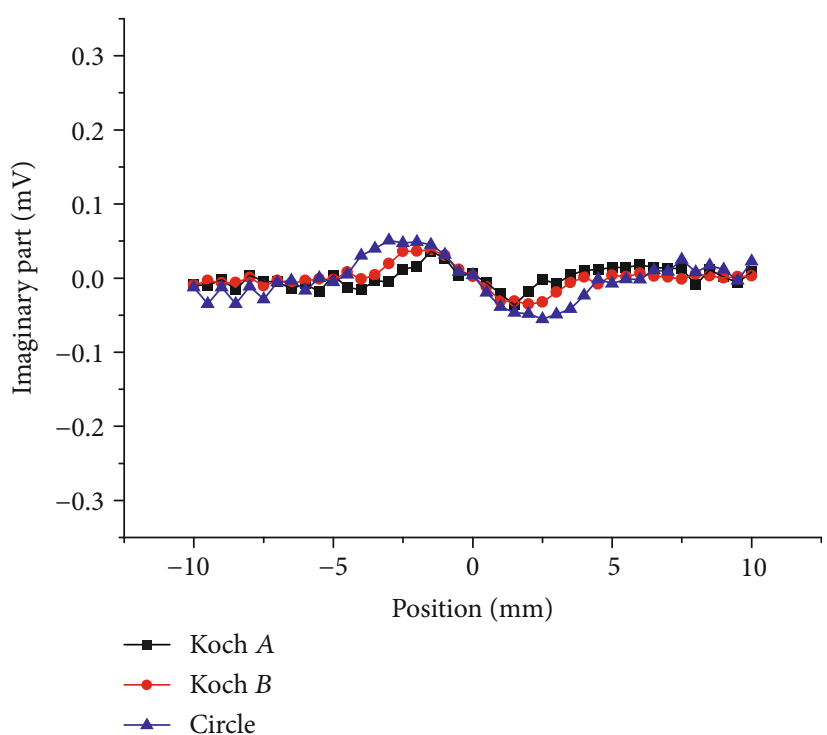

(b)

FIGURE 7: The signals for the infinite defect: (a) real part; (b) imaginary part.

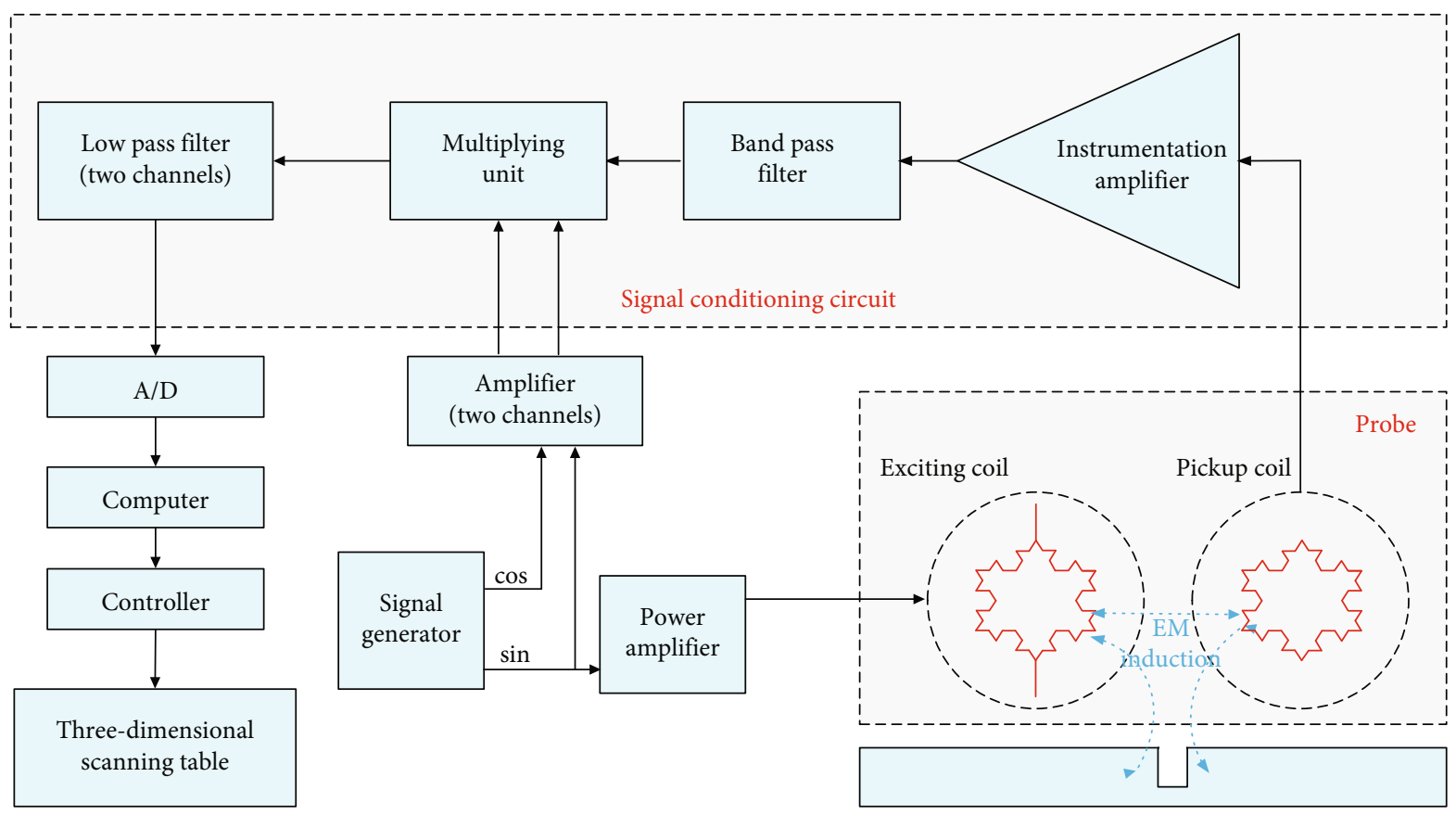

FIgURE 8: The schematic diagram of the experiment system.

The mesh of every model is automatically divided by the extra fine element size in the software. The frequency of each model is $100 \mathrm{kHz}$. If the specimen in the model has an infinite defect, the defect will move from $-10 \mathrm{~mm}$ to $10 \mathrm{~mm}$ in a step of $0.5 \mathrm{~mm}$. Then, the output signal is as follows [25]:

$$
V=\omega \oint \mathbf{A} \cdot d \mathbf{l}
$$

where $V$ is the output signal of the pick-up coil and $d \mathbf{l}$ is the infinitesimal vector of the pick-up coils.

The ECs on the upper surface of the perfect specimen are shown in Figure 6, and the output signals for the specimens with the infinite defect are shown in Figure 7. In Figure 6, every EC distribution is similar to the shape of the exciting coil. In Figure 7, for each probe, the trend of the waveforms of the signals is similar, but the real parts and the imaginary parts are in antiphase. The $V_{\mathrm{pp}}$ (peak-peak value) of the circular probe is slightly larger than that of another two probes. 


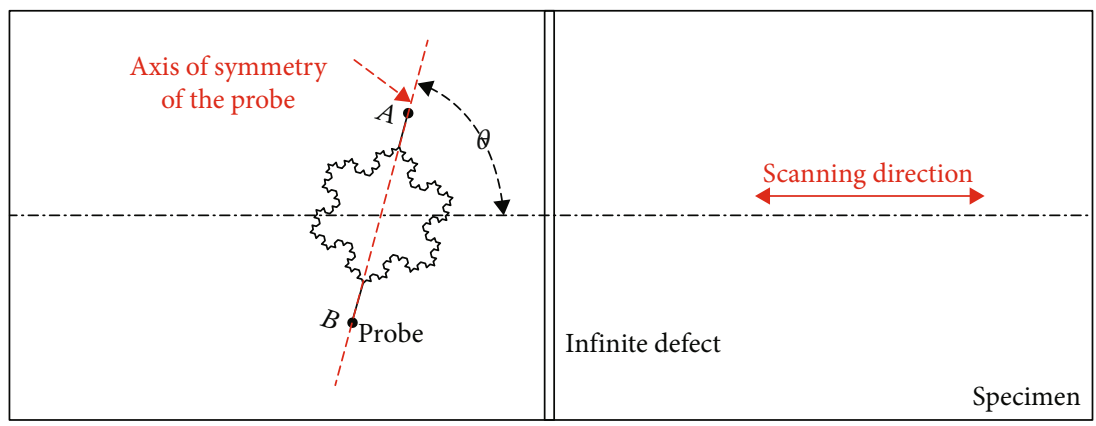

FIgURE 9: The diagram for the sensitive detection of the probes.

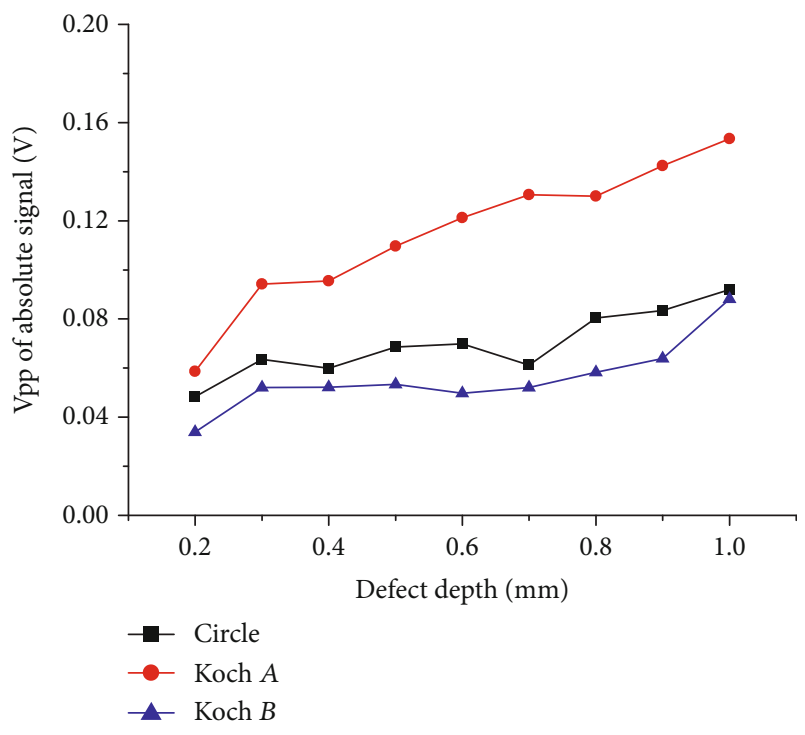

Figure 10: $V_{\mathrm{pp}}$ of the absolute signal of the different depth defects.

\section{Experiment}

The schematic diagram of the experiment system is shown in Figure 8. This system is identical to that in the literature [26] with the exception of the number of exciting channels. The number of exciting channels in this system is only one. In this work, the root mean square and frequency of the exciting current are $0.2 \mathrm{~A}$ RMS and $100 \mathrm{kHz}$, respectively.

There are two sets in the experiment. The first set is conducted to find the sensitive axis of the probes. The specimen with size $350 \mathrm{~mm} \times 80 \mathrm{~mm} \times 6 \mathrm{~mm}$ in this experiment has an infinite defect whose size is $0.25 \mathrm{~mm} \times 80 \mathrm{~mm} \times 3 \mathrm{~mm}$. The probes move on the upper surface of the specimen along the scanning direction. In this experiment, the angle $\theta$ between the $A B$ axis of symmetry of the probes and the scanning directions is changed from $0^{\circ}$ to $90^{\circ}$ with the step of $30^{\circ}$. This experiment is conducted to find the sensitive direction, as shown in Figure 9. The direction in which the $V_{\mathrm{pp}}$ of the signal has the maximum values will be selected to be the direction of the probes. For the second set of the experiment, the probes will move along the sensitive direction.

For the second set of experiments, the defect detection performances of the three probes are compared by sensing defects with different lengths and depths and in different directions. For the different length defect detection, the lengths of the defects are $20 \mathrm{~mm}, 15 \mathrm{~mm}, 10 \mathrm{~mm}, 5 \mathrm{~mm}$, $3 \mathrm{~mm}$, and $1 \mathrm{~mm}$, respectively; for the different depth defect detection, the depths are changed from $0.2 \mathrm{~mm}$ to $1 \mathrm{~mm}$ with the step of $0.1 \mathrm{~mm}$; for the different direction defect detection, the angle between the sensitive axis of the probes and the defect direction is changed from $0^{\circ}$ to $90^{\circ}$ with the step of $15^{\circ}$, the defect sizes are $5 \mathrm{~mm} \times 1 \mathrm{~mm} \times 0.22 \mathrm{~mm}$ and 15 $\mathrm{mm} \times 1 \mathrm{~mm} \times 0.22 \mathrm{~mm}$, and the results of this experiment are transformed into $180^{\circ}$ by using symmetry as shown in Figure 10. The widths of all defects in this set experiment are $0.22 \mathrm{~mm}$.

\section{Results and Discussion}

5.1. Experiment for Finding the Sensitive Direction of the Three Probes. As can be seen in Figure 11, there are the experimental results of the three probes in definite directions. In the scanning results of each probe which is at different angles for infinite length defects, the $V_{\mathrm{pp}}$ of the real parts and imaginary parts of the output signals from the probes are larger than the $V_{\mathrm{pp}}$ at other angles while the angle between the axis of the probe and the scanning axis is $90^{\circ}$, as shown in Figure 9. Therefore, in all subsequent experiments, the $A B$ axes of probes are all perpendicular to scanning directions in the scanning process.

At the results in Figure 11, when the angle between the axis of every probe and the scanning direction is $90^{\circ}$, the real parts and imaginary parts of output signals from every kind of probe all have a peak value and a valley value, and the real parts and imaginary parts are in antiphase. For the real parts and the imaginary parts of defect output signals from the three kinds of probes, the $V_{\mathrm{pp}}$ of the output signals from the circular probe is the largest in the same detective condition. There are three reasons that can explain this result: the defect sizes adopted in the experiment are much larger than the size of the probes; all the three kinds of probes are of a considerably strong EC disturbance; the area of the signal pick-up coil of the circular probe is larger than that of the other two probes. Thus, the defect output signals from the signal pick-up coil of the circular probe is the strongest. However, can this result indicate that the sensitivity of the circular probe is higher than the other two kinds of Koch probes for short defect detecting? For the defects with the 


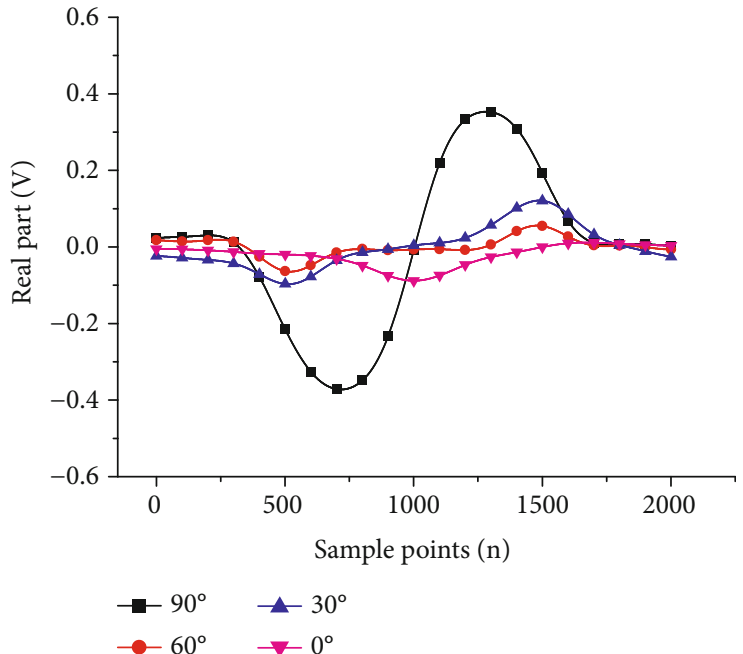

(a)

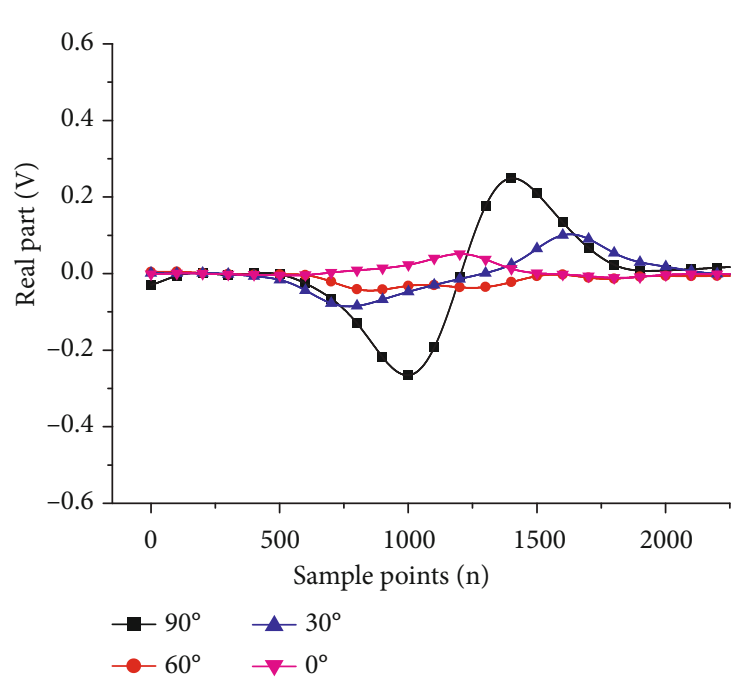

(c)

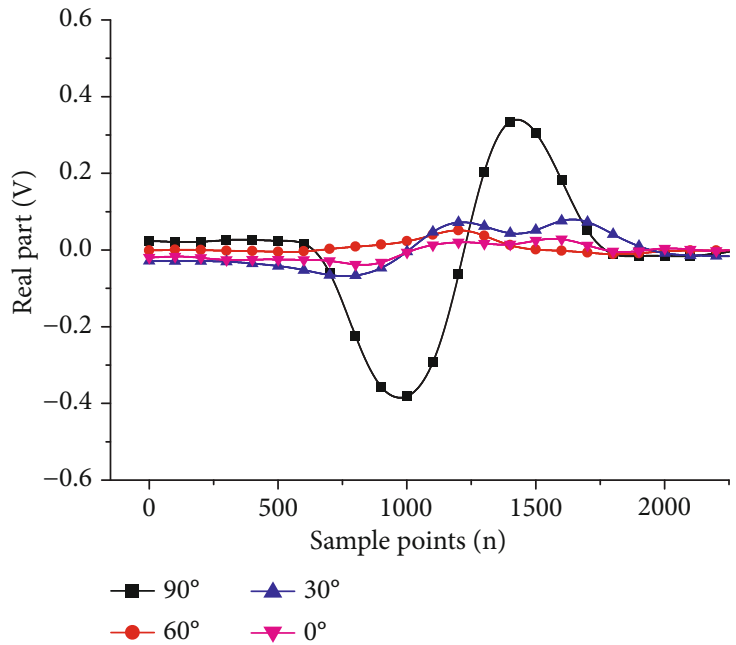

(e)

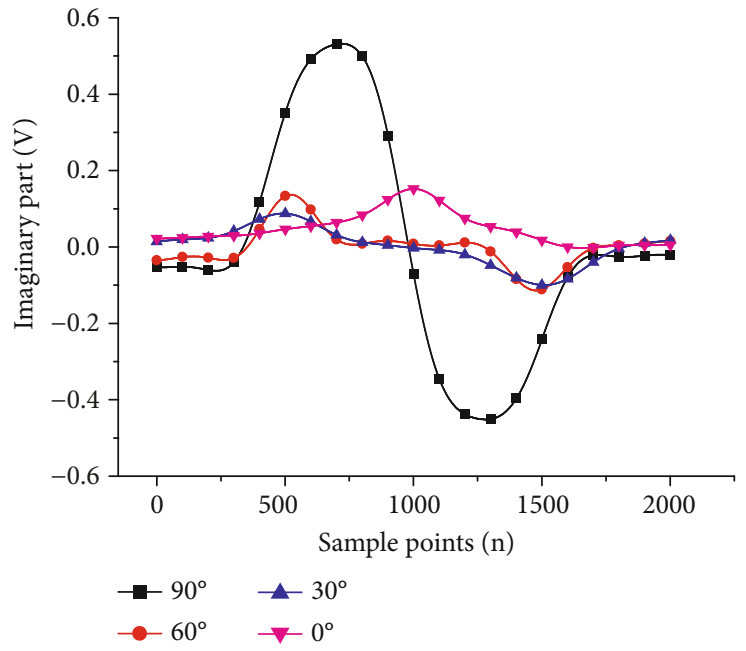

(b)

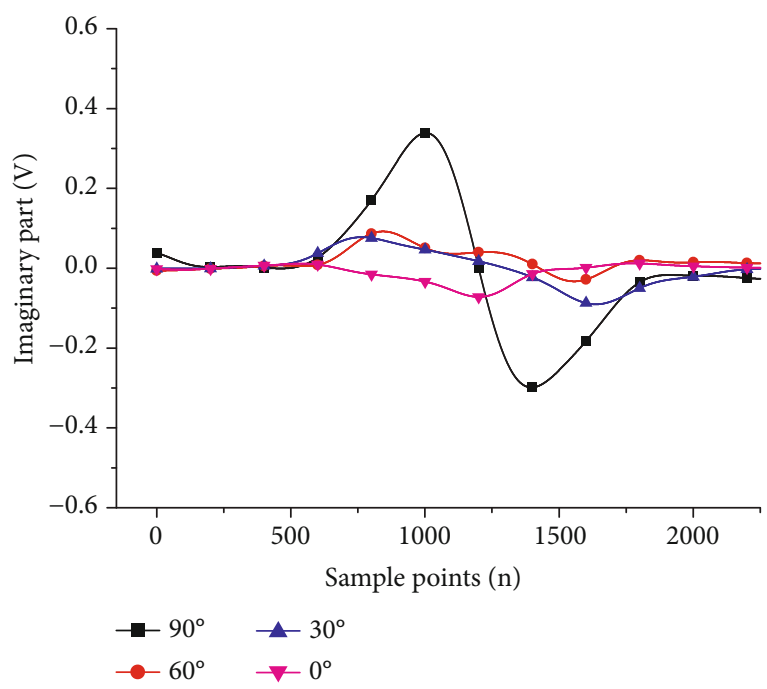

(d)

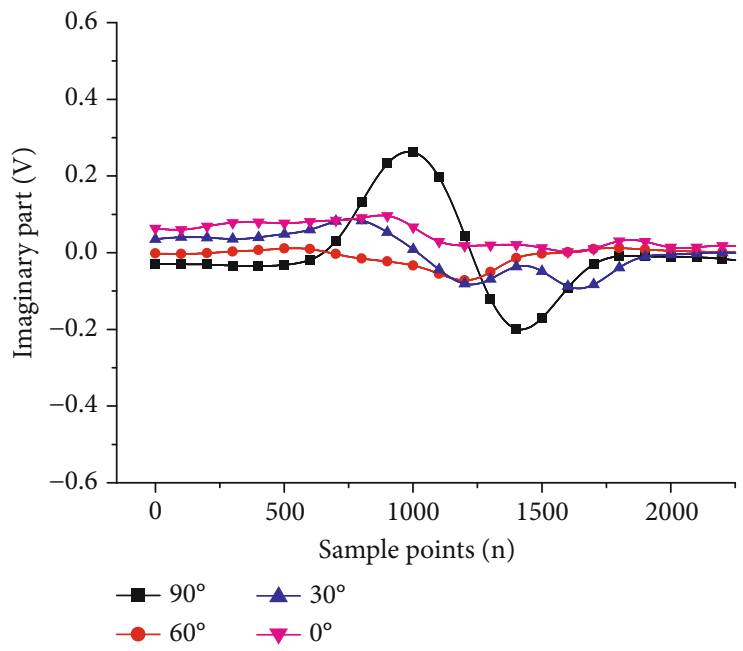

(f)

FIGURE 11: Experiment result for finding the sensitive axis of the three probes: (a) real part of the circular probe; (b) imaginary part of the circular probe; (c) real part of Koch A probe; (d) imaginary part of Koch A probe; (e) real part of Koch $B$ probe; (f) imaginary part of Koch $B$ probe. 
TABLe 1: $V_{\mathrm{pp}}$ of the real part and imaginary part for the different length defect detection.

\begin{tabular}{lccccccccc}
\hline \multirow{2}{*}{ Defect length $(\mathrm{mm})$} & Circle & Real part $(\mathrm{V})$ & \multicolumn{3}{c}{ Imaginary part $(\mathrm{V})$} & \multicolumn{3}{c}{ Absolute $(\mathrm{V})$} \\
& 0.423 & 0.299 & 0.270 & 0.190 & 0.259 & 0.189 & 0.238 & 0.209 & 0.168 \\
20 & 0.459 & 0.350 & 0.308 & 0.215 & 0.337 & 0.233 & 0.246 & 0.254 & 0.190 \\
15 & 0.359 & 0.301 & 0.303 & 0.211 & 0.324 & 0.250 & 0.205 & 0.227 & 0.189 \\
10 & 0.144 & 0.114 & 0.098 & 0.081 & 0.259 & 0.086 & 0.088 & 0.150 & 0.066 \\
5 & 0.045 & 0.041 & 0.021 & 0.053 & 0.091 & 0.018 & 0.029 & 0.056 & 0.014 \\
3 & 0.029 & 0.025 & 0.023 & 0.048 & 0.050 & 0.010 & 0.015 & 0.031 & 0.011 \\
1 & & & & & & & & Koch $B$ & \\
\hline
\end{tabular}

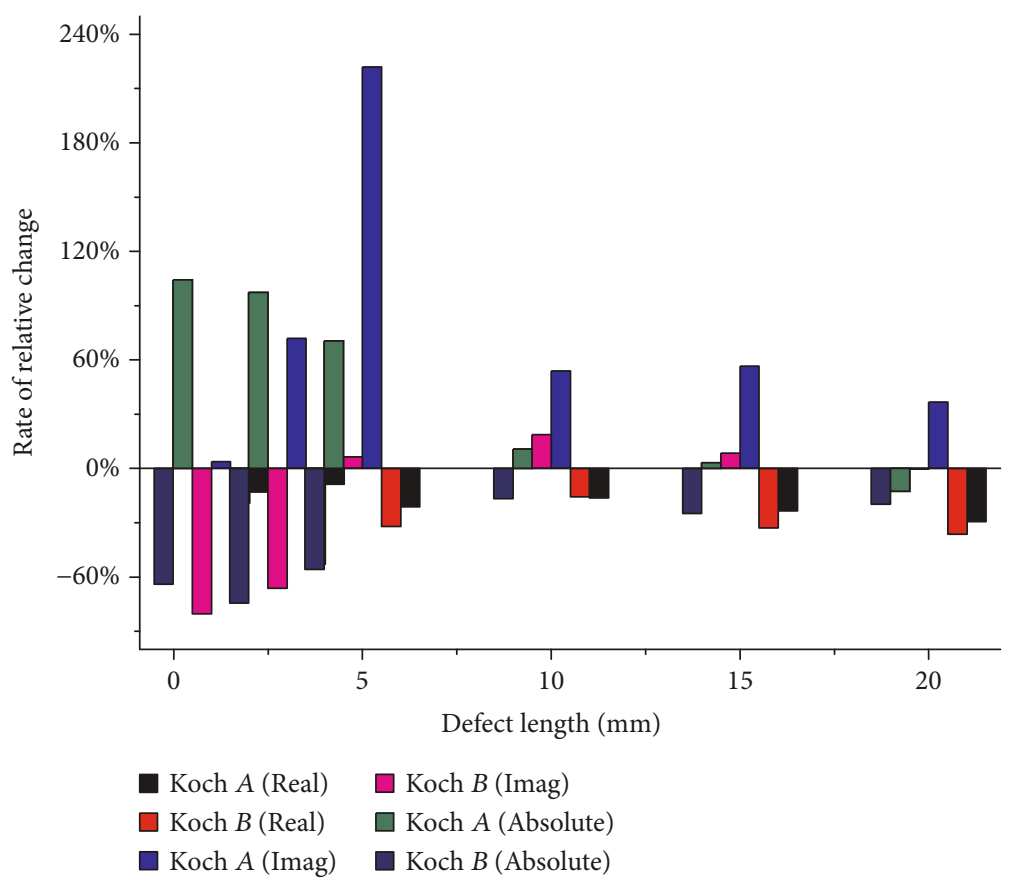

FIgURE 12: The rate of relative change of the $V_{\mathrm{pp}}$ of the signals to the circular probe.

relatively large sizes, for instance, the experiment shows that all the defects have a strong disturbance to the EC, which does not present the edge of the Koch curve: it can adjust the EC distribution. Instead, the area of the signal pick-up coil of the Koch curves becomes smaller, which results in the output defect signals that are relatively weak. For the defects with the relatively small sizes, an experiment is indispensable to prove which one has higher sensitivity between the three kinds of probes because the EC disturbances induced by the three kinds of probes in short size defects are different.

5.2. Experiment for Different Length Defect Detection. The three kinds of probes used and the $V_{\mathrm{pp}}$ of the real parts and imaginary parts for the different length defect detection are presented in Table 1. For the real part signals, the $V_{\mathrm{pp}}$ of the real part signal output from the circular probe are larger than those of the output from the other two kinds of probes when the defect length is larger than $5 \mathrm{~mm}$; when the defect length is less than or equal to $5 \mathrm{~mm}$, the $V_{\mathrm{pp}}$ of the real part signals of the Koch A probe are close to those of the circular probe, while the $V_{\mathrm{pp}}$ of the real part signals of the Koch $B$ probe are all less than those of the output from the circular probe. As for the imaginary part signals, in all the different length defects detected, the $V_{\mathrm{pp}}$ of the imaginary part signals of the Koch A probe are all larger than those of the other two kinds of probes. For the $V_{\mathrm{pp}}$ of the absolute signal, for each length defect, that of the Koch $B$ probe is less than that of the circular probe; but except for the $20 \mathrm{~mm}$ length defect, that of the Koch A probe is larger than that of the circular probe.

In order to research the difference of the output signals between the two kinds of Koch probes and the circular probe, for every signal of every length defect, the rate of relative change of the $V_{\mathrm{pp}}$ of the signals to the circular probe is calculated. It can be formulated as follows:

Rate of relative change $=\frac{V_{\mathrm{pp}} \text { of Koch probe }-V_{\mathrm{pp}} \text { of circular probe }}{V_{\mathrm{pp}} \text { of circular probe }}$. 


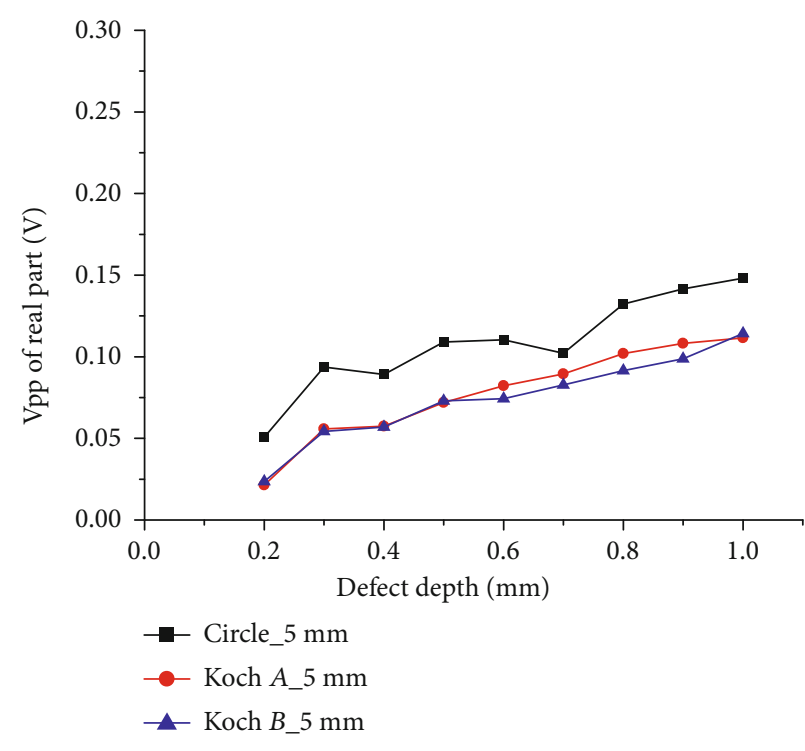

(a)

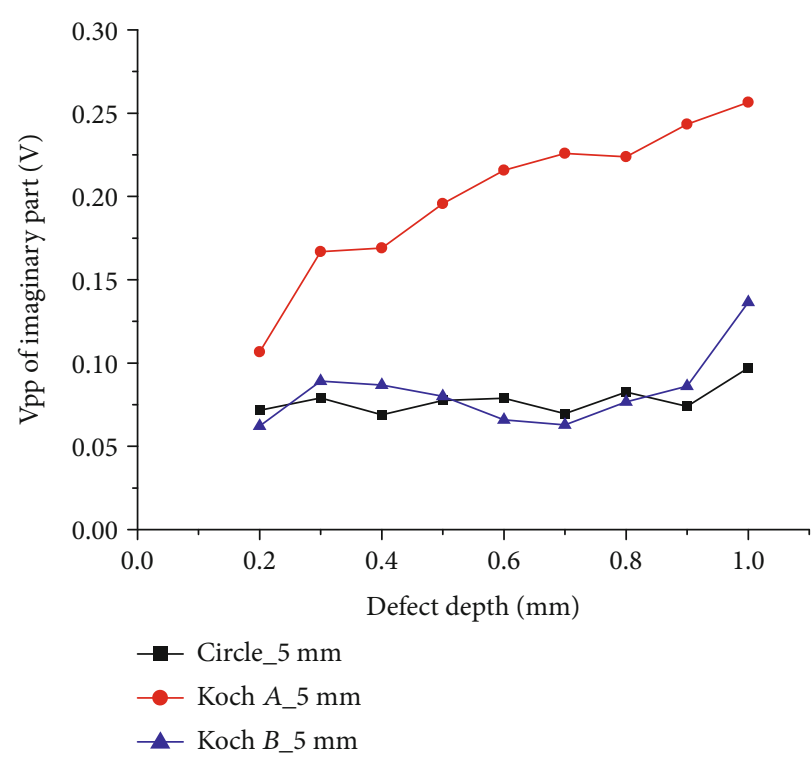

(b)

FIGURE 13: Experiment result for different depth defect detection: (a) $V_{\text {pp }}$ of real part of the three kinds of probes; (b) $V_{\text {pp }}$ of imaginary part of the three kinds of probes.

The calculation results are shown in Figure 12. For the defects with $20 \mathrm{~mm}, 15 \mathrm{~mm}, 10 \mathrm{~mm}, 5 \mathrm{~mm}, 3 \mathrm{~mm}$, and $1 \mathrm{~mm}$ lengths, the rates of relative change of the real parts of the Koch A probe are $-29 \%,-24 \%,-16 \%,-21 \%,-9 \%$, and $-13 \%$, respectively, and the rates of relative change of the imaginary parts are $37 \%, 57 \%, 54 \%, 222 \%, 72 \%$, and $4 \%$, respectively; the rates of relative change of the real parts of the Koch $B$ probe are $-36 \%,-33 \%,-16 \%,-32 \%,-53 \%$, and $-19 \%$, respectively, and the rates of relative change of the imaginary parts are $0 \%,-8 \%, 18 \%, 6 \%,-66 \%$, and $-80 \%$, respectively; the rates of relative change of the absolute signals of Koch A are $-12 \%, 3 \%, 20 \%, 70 \%, 97 \%$, and 104\%, respectively, and that of Koch $B$ are $-19 \%,-24 \%,-16 \%$, $-55 \%,-74 \%$, and $-63 \%$, respectively.

From what has been discussed above, it can be concluded that the Koch A probe is of higher sensitivity, when detecting the defects with $5 \mathrm{~mm}$ and $3 \mathrm{~mm}$ lengths which are shorter than the size of the probes. For the Koch $B$ probe, with the $V_{\mathrm{pp}}$ of the real parts and the imaginary parts relative to those of the circular probe considered, it has no obvious advantages. Although the Koch curves are used as the exciting coils, what causes the difference between the Koch A probe and the Koch $B$ probe relative to the circular probe could be the position of the Koch curves in the two kinds of probes. The Koch curve along with the midperpendicular of the $A B$ axis is convex in the Koch A probe and is concave in the Koch $B$ probe.

5.3. Experiment for Different Depth Defect Detection. As is shown in Figure 13, there are the $V_{\mathrm{pp}}$ of the real parts and the imaginary parts to the detection signal of different depth defects with $5 \mathrm{~mm}$ length about the three kinds of probes. For each probe, the $V_{\mathrm{pp}}$ of the signals increase with the increase of the defect depth. For the real parts, the $V_{\mathrm{pp}}$ of the two kinds of Koch probes are less than those of the circular probe; for the imaginary parts, the $V_{\mathrm{pp}}$ of the Koch A probe are all larger than those of the other two kinds of probes. While the defect depth increases to $1 \mathrm{~mm}$, the $V_{\mathrm{pp}}$ of the output signals of the Koch A probe is approximately $0.26 \mathrm{~V}$, and that of the circular probe and the Koch $B$ probe is $0.10 \mathrm{~V}$ and $0.13 \mathrm{~V}$, respectively. In this case, the $V_{\mathrm{pp}}$ in the real parts of the output signals of the Koch A probe is 2.6 times larger and twice larger than the circular probe and the Koch $B$ probe, respectively.

Figure 10 shows the $V_{\mathrm{pp}}$ signal of the absolute signal of the different depth defects, which is calculated from the result in Figure 13. For all depths of the defects, the $V_{\mathrm{pp}}$ value of the absolute signal is stronger than that of the Koch $B$ and circular probes. When the depth of the defect is $0.2 \mathrm{~mm}$, the values of the Koch A, Koch B, and circular probe are about $0.06 \mathrm{~V}$, $0.03 \mathrm{~V}$, and $0.05 \mathrm{~V}$, respectively; When the depth of the defect is $1.0 \mathrm{~mm}$, the values of the Koch $A$, Koch $B$, and circular probe are $0.15 \mathrm{~V}, 0.08 \mathrm{~V}$, and $0.09 \mathrm{~V}$, respectively. Therefore, the Koch A probe's performance for detecting the $5 \mathrm{~mm}$ length defect in hard detection direction is superior to the other two probes.

In Figures 13 and 10, the performance of the Koch $\mathrm{A}$ probe is better than that of the circular probe, because the Koch curve can induce the eddy current in the specimen distributed in more directions in a smaller space domain, and the eddy current can be better disturbed by the short defect which is perpendicular to the scanning direction. Then, why the performance of the Koch A probe excels that of Koch $B$ probe may be because of the eddy current distributions induced by the two probes [22]. In the scanning direction, the eddy current distribution of the Koch A probe is convex to external, but the eddy current distribution of the Koch $B$ probe is invaginated internally. The angle between the eddy current and the defect of Koch A is more than that of the 


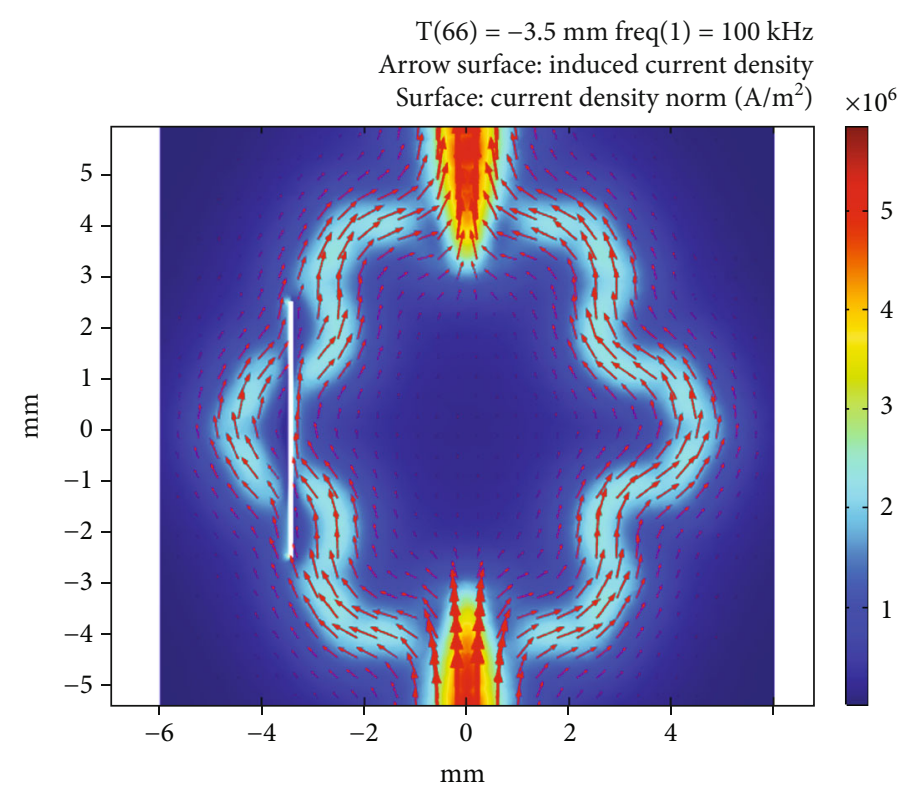

(a)

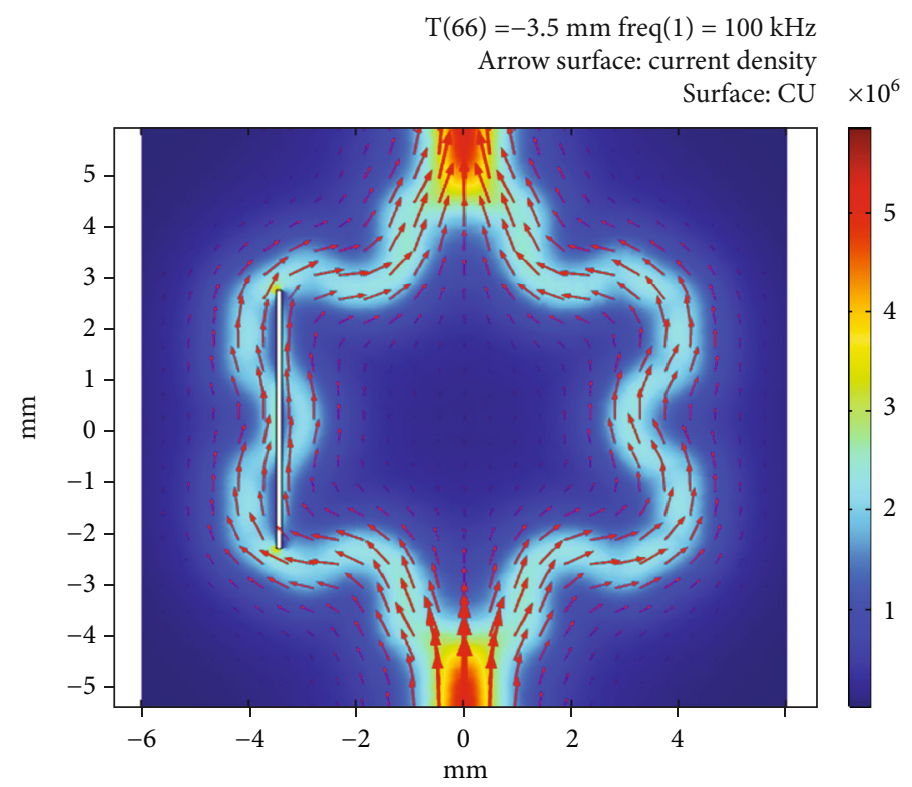

(b)

Figure 14: Eddy current distribution of the two probes disturbed by the $5 \mathrm{~mm}$ length defect: (a) Koch A; (b) Koch $B$.

Koch $B$ probe. This may be the cause of the above phenomenon. The arrow and magnitude of the eddy current distribution disturbed by $5 \mathrm{~mm}$ length defect at the maximum output signal position are illustrated in Figure 14.

5.4. Experiment for Different Direction Defect Detection. The $V_{\mathrm{pp}}$ of the real parts and imaginary parts in the scanning signals of the three kinds of probes for the different direction defects with $5 \mathrm{~mm}$ and $1 \mathrm{~mm}$ length are shown in Figure 15. In Figure 15(a) are the $V_{\mathrm{pp}}$ of the real part signals of the three kinds of probes for the defect detection with $5 \mathrm{~mm}$ length. The $V_{\mathrm{pp}}$ of the real parts of the signal output from every kind of probe are all the smallest when the defect is at $90^{\circ}$; as for a defect with a certain angle, there is little difference among the $V_{\mathrm{pp}}$ of the real parts of the output signals from the three kinds of probes. In Figure 15(b) are the $V_{\mathrm{pp}}$ of the imaginary part signals for the $5 \mathrm{~mm}$ defect detection scanned by the three kinds of probes. The $V_{\mathrm{pp}}$ of the output signals from the Koch $B$ probe is the smallest when the defect is at $90^{\circ}$, while the $V_{\mathrm{pp}}$ of the Koch A probe is much larger than that of the output signal of the other two kinds of probes. There are the $V_{\mathrm{pp}}$ of the real parts of the detection signals from the three kinds of probes relative to $10 \mathrm{~mm}$ defect in different directions as shown in Figure 15(c). Compared to the other two kinds of probes, the circular probe has the advantages in every direction. As is shown in Figure 15(d), there are 


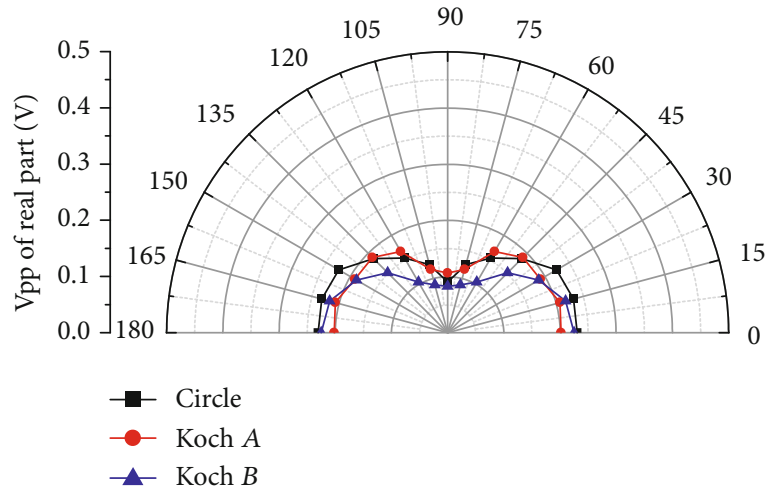

(a)

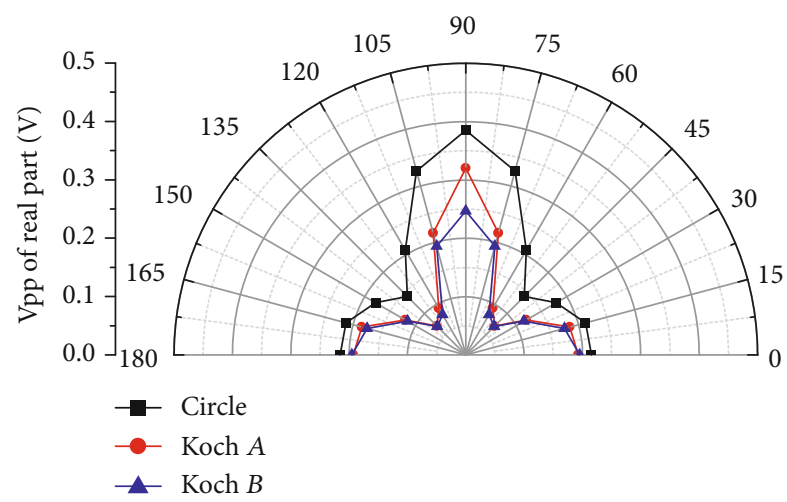

(c)

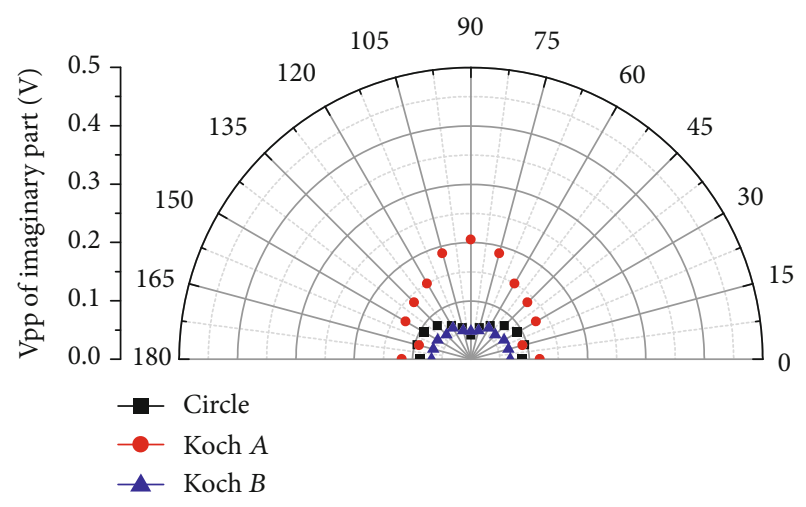

(b)

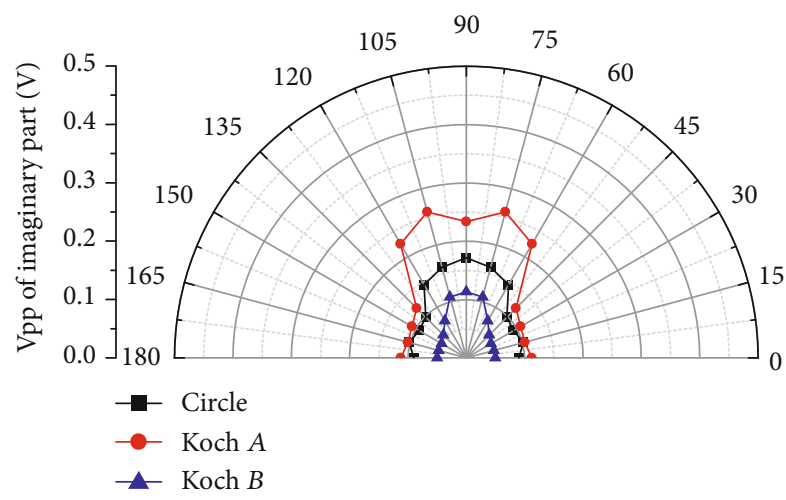

(d)

FIGURE 15: Experiment result for different direction defect detection: (a) $V_{\text {pp }}$ of real part of the three kinds of probes for 5 mm defect detection; (b) $V_{\mathrm{pp}}$ of imaginary part of the three kinds of probes for $5 \mathrm{~mm}$ defect detection; (c) $V_{\mathrm{pp}}$ of real part of the three kinds of probes for $10 \mathrm{~mm}$ defect detection; (d) $V_{\mathrm{pp}}$ of imaginary part of the three kinds of probes for $10 \mathrm{~mm}$ defect detection.

the $V_{\mathrm{pp}}$ of the imaginary parts of the detection signals from the three kinds of probes relative to $10 \mathrm{~mm}$ defect in different directions. When the defect direction is between $45^{\circ}$ and $135^{\circ}$, the $V_{\mathrm{pp}}$ of the output signals from the Koch A probe are all larger than those of the other two kinds of probes and are comparatively close to those of the circular probe at other angles.

\section{Conclusions}

In this study, the method of the coil connection of the differential exciting EC probe has been abstracted to two kinds of topological structures, the series-wound and parallel-wound topological structure, via the geometric transformation method of topology. This study made Koch curves combine with the parallel-wound topological structure; then, the two kinds of Koch EC probes with parallel-wound topological structure were proposed and designed. In order to verify the adjustment effect of the Koch coil which is relative to the circular coil for the EC in spatial distribution, a kind of circular EC probe that possesses a parallel-wound topological structure was designed. The EC distributions of the three kinds of probes on the surface of the aluminum sheets and the signals to infinite length defects were obtained by the FEM simulation. It is confirmed that the sensitive directions of the three kinds of probes are all the midperpendiculars of the $A B$ axes by detecting the infinite length defects. From the experimental results for detecting the defects with different lengths, depths, and directions, it can be concluded that the sensitivity of the Koch A EC probe which has a serieswound topological structure is higher than that of the circular coil when the defect in length is shorter than the size of coils of the probe.

The advantages of the proposed probe are as follows:

(i) It can detect the short defects in the special direction, but the same-sized circular coil detects the defects difficultly

(ii) It can be designed on a flexible PCB to detect the flaws in the conductive parts with the complex surfaces

(iii) It is a differential exciting eddy current probe, so it can suppress the lift-off noise by the coil structure design

The disadvantage of the proposed probe is that the defect signal is very weak and carries strong noise because the exciting and pick-up coils are only one turn, respectively.

In the future, the lift-off effect and the $\mathrm{C}$ scan image of the Koch A probe will be deeply studied. 


\section{Data Availability}

The signal data used to support the findings of this study have not been made available because of secrecy.

\section{Conflicts of Interest}

The authors declare that they have no conflict of interest.

\section{Acknowledgments}

This work was financially supported by the National Natural Science Foundation of China (Grant No. 51807086).

\section{References}

[1] G. Antonelli, F. Binasco, G. Danese, and D. Dotti, "Virtually zero cross-talk dual-frequency eddy current analyzer based on personal computer," IEEE Transactions on Instrumentation and Measurement, vol. 43, no. 3, pp. 463-468, 1994.

[2] A. Bernieri, G. Betta, L. Ferrigno, and M. Laracca, "Crack depth estimation by using a multi-frequency ECT method," IEEE Transactions on Instrumentation and Measurement, vol. 62, no. 3, pp. 544-552, 2013.

[3] G. Betta, L. Ferrigno, M. Laracca, P. Burrascano, M. Ricci, and G. Silipigni, "An experimental comparison of multi-frequency and chirp excitations for eddy current testing on thin defects," Measurement, vol. 63, pp. 207-220, 2015.

[4] A. Sophian, G. Tian, and M. Fan, "Pulsed eddy current nondestructive testing and evaluation: a review," Chinese Journal of Mechanical Engineering, vol. 30, no. 3, pp. 500-514, 2017.

[5] L. Yong, Y. Bei, W. Li, H. Jing, Z. Chen, and L. Da, "Pulsemodulation eddy current probes for imaging of external corrosion in nonmagnetic pipes," NDT \& E International, vol. 88, pp. 51-58, 2017.

[6] P. Li, S. Xie, K. Wang et al., "A novel frequency-band-selecting pulsed eddy current testing method for the detection of a certain depth range of defects," NDT \& E International, vol. 107, p. 102154, 2019.

[7] S. Xie, L. Zhang, Y. Zhao et al., "Features extraction and discussion in a novel frequency-band-selecting pulsed eddy current testing method for the detection of a certain depth range of defects," Ndt \& E International, vol. 111, p. 102211, 2020.

[8] S. Li, S. Huang, and W. Zhao, "Development of differential probes in pulsed eddy current testing for noise suppression," Sensors and Actuators A: Physical, vol. 135, pp. 675-679, 2007.

[9] L. S. Rosado, T. G. Santos, P. M. Ramos, P. Vilaça, and M. Piedade, "A new dual driver planar eddy current probe with dynamically controlled induction pattern," NDT \& $E$ International, vol. 70, pp. 29-37, 2015.

[10] C. Ye, Y. Huang, L. Udpa, and S. S. Udpa, "Novel rotating current probe with GMR array sensors for steam generate tube inspection," IEEE Sensors Journal, vol. 16, no. 12, pp. 49955002, 2016.

[11] G. Dib, G. Yang, C. Ye, A. Tamburrino, L. Udpa, and S. S. Udpa, "EC-GMR array with rotating current excitation for multilayered riveted structures inspection," in Presented at Aip Conference, 2015.

[12] K. Toshihiko, "Small eddy current testing sensor probe using a tunneling magnetoresistance sensor to detect cracks in steel structures," IEEE Transactions on Magnetics, vol. 54, pp. 1-5, 2018.
[13] T. S. Santos, P. M. Ramos, and P. S. Vilaça, "Non destructive testing of friction stir welding: comparison of planar eddy current probes," Presented at Imeko Tc4 Symposium, Florence, Italy, 2008.

[14] X. Peng and J. Huang, "A new eddy current sensor composed of three circumferential gradient winding coils," in Presented at Seventh International Conference on Sensing Technology, Wellington, New Zealand, 2013.

[15] Z. Sun, D. Cai, C. Zou, W. Zhang, and Q. Chen, "Design and optimization of a flexible arrayed eddy current sensor," Measurement Science \& Technology, vol. 28, no. 4, article 045105, 2017.

[16] Z. Sun, D. Cai, C. Zou, W. Zhang, and Q. Chen, “A flexible arrayed eddy current sensor for inspection of hollow axle inner surfaces," Sensors, vol. 16, no. 7, p. 952, 2016.

[17] N. J. Goldfine, Uncalibrated, Absolute Property Estimation and Measurement Optimization for Conducting and Magnetic Media Using Imposed [Omega]-k Magnetometry, Massachusetts Institute of Technology, 1991.

[18] L. U. Daura, G. Y. Tian, Q. J. Yi, and A. Sophian, "Wireless power transfer-based eddy current non-destructive testing using a flexible printed coil array," Philosophical Transactions of the Royal Society A: Mathematical, Physical and Engineering Sciences, vol. 378, no. 2182, 2020.

[19] S. B. She, Y. Z. He, Y. F. Chen, and T. Chady, "Flexible floral eddy current probe for detecting flaws in metal plate," IEEE Sensors Journal, vol. 20, no. 18, pp. 10521-10529, 2020.

[20] N. Zhang, C. Ye, L. Peng, and Y. Tao, "Novel array eddy current sensor with three-phase excitation," IEEE Sensors Journal, vol. 19, no. 18, pp. 7896-7905, 2019.

[21] S. Khan, L. Lorenzelli, and R. S. Dahiya, "Technologies for printing sensors and electronics over large flexible substrates: a review," IEEE Sensors Journal, vol. 15, no. 6, pp. 3164$3185,2015$.

[22] G. Chen, W. Zhang, and W. Pang, "Koch curve fractal geometry excitation probe for eddy current non-destructive testing," Measurement, vol. 124, pp. 470-478, 2018.

[23] G. L. Chen, W. M. Zhang, X. Y. Gao, and X. Yu, "A finite element study of the differential pick-up coil and the differential excitation coil for eddy current ndt," in In Electromagnetic nondestructive evaluation 40, Studies in Applied Electromagnetics and Mechanics, Z. Chen, S. Xie, and Y. Li, Eds., pp. 133-140, Ios Press, Amsterdam, 2015.

[24] K. Ueno, K. Shiga, and S. Morita, A Mathematical Gift, I, II, III: The Interplay between Topology, Functions, Geometry, and Algebra [M], 2005.

[25] G. Chen, W. Zhang, Z. Zhang, X. Jin, and W. Pang, "A new rosette-like eddy current array sensor with high sensitivity for fatigue defect around bolt hole in SHM," NDT \& E International, vol. 94, pp. 70-78, 2018.

[26] G. Chen, W. Zhang, W. Jin et al., "A novel rotational field eddy current planar probe with two-circular sector pickup coils," Sensors, vol. 19, no. 21, p. 4628, 2019. 